\title{
Transverse beam resonance in the superconducting linac of the Spallation Neutron Source
}

\author{
Y. Zhang, ${ }^{*}$ C. K. Allen, J. D. Galambos, J. Holmes, and J. G. Wang \\ Oak Ridge National Laboratory, Oak Ridge, Tennessee 37831, USA
}

(Received 27 August 2009; published 19 April 2010)

\begin{abstract}
A weak transverse resonance in the Spallation Neutron Source (SNS) superconducting linac is identified in computer simulations, and is believed to be one of the mechanisms behind measured beam losses. This weak resonance is induced by the nonlinear dodecapole component of the linac quadrupole magnets. It occurs when the linac focusing lattice has a transverse phase advance close to $60^{\circ}$. By reducing the phase advance to approximately $50^{\circ}$ to avoid the resonance, we observe significant reduction in beam loss in the SNS superconducting linac. We present theory and computer simulation results supporting the notion that a suppression of the $60^{\circ}$ resonance may contribute to reduction in the beam loss.
\end{abstract}

DOI: 10.1103/PhysRevSTAB.13.044401

PACS numbers: 29.20.Ej, 29.27.Bd

\section{INTRODUCTION}

The Spallation Neutron Source (SNS) is a short-pulse neutron scattering facility at Oak Ridge National Laboratory. The SNS accelerator complex consists of a $1 \mathrm{GeV} \mathrm{H}^{-}$linear accelerator, an accumulator ring, and associated beam transport lines [1]. The linear accelerator includes an injector, a $2.5 \mathrm{MeV}$ radio-frequency quadrupole (RFQ), a medium energy beam transport (MEBT) line, a drift tube linac (DTL), a coupled cavity linac (CCL) for beam energies up to $186 \mathrm{MeV}$, and a superconducting linac (SCL) for energies up to $1 \mathrm{GeV}$. The linac design lattice is shown in Fig. 1.

The total length of the SNS linac is approximately $330 \mathrm{~m}$ : the normal conducting linac has mainly a periodic lattice of focusing quadrupole (F), drift space (O), defocusing quadrupole (D), and drift space (O) (FODO) lattice and is about $100 \mathrm{~m}$ long; the superconducting linac has a doublet focusing lattice and is about $230 \mathrm{~m}$ long, including a $70 \mathrm{~m}$ dummy section at the end for future upgrade. In the baseline design, a $1.56 \mathrm{MW} \mathrm{H}^{-}$beam is delivered through the linac. Minimizing beam loss and residual activation is critical to the operation of this high-power accelerator. The situation is particularly severe in the SCL because a beam loss above $1 \mathrm{~W} / \mathrm{m}$ may quench superconducting cavities, and interrupt normal operation. In the design, the expected fractional beam loss in the SCL is less than $1 \times 10^{-5}$; but based on residual activations and from beam loss measurement, the total fractional loss was estimated to be much larger than this value. From October 2006 to April 2009 the SNS beam power was ramped up from 10 to $860 \mathrm{~kW} \mathrm{[2],}$ beam loss and residual activation in the linac were increased as the beam power.

Recently, we analyzed the imperfections of the linac quadrupole magnets, which mainly include dodecapole

\footnotetext{
*Present address: Research Accelerator Division, SNS, Oak Ridge National Laboratory, P.O. Box 2008, MS 6461, Oak Ridge, TN 37831-6461.
}

components. These magnets contain dipole correctors formed from additional windings that induce, additionally, a sextupole component. From simulation studies and beam loss measurements, we found that the SCL design lattice has a weak resonance at a phase advance of $60^{\circ}$. The dodecapole terms of the linac quadrupoles are responsible for generating this resonance and a small fraction of the beam particles are lost in the SCL due to the nonlinear forces. This weak resonance is not driven by space-charge. It appears only when the contributions of dodecapoles are significant, and when the linac lattice has a transverse phase advance close to $60^{\circ}$. After we reduced the linac quadrupole strengths to a new phase advance of approximately $50^{\circ}$, beam loss in the linac decreased significantly, especially in the second half of the SCL [3].

Beam loss in the linac is a complex problem; for example, the SNS loss levels of $10^{-5}$ to $10^{-4}$ are beyond any direct beam current measurement, or even the accuracy of computer models. To investigate the mechanisms of the SCL loss reduction with the reduced quadrupole strengths, we design a lattice having a $70^{\circ}$ phase advance to compare the effects of several loss mechanisms, such as longitudinal halo, magnetic field stripping of $\mathrm{H}^{-}$beams, transverse matching conditions, as well as other nonlinearity issues. The $70^{\circ}$ phase advance lattice cannot completely avoid the $60^{\circ}$ resonance because of space-charge tune depressions. In addition, because of the increased quadrupole field strengths, the absolute value of all higher-order multipole field strengths is also increased. Even so, simulation and measurement results show that beam loss decreases slightly in this case.

\section{BEAM EMITTANCE GROWTH}

There are a total of 24 FODO cells in the CCL and 32 doublet cells in the SCL, including the dummy section. The cell-by-cell, zero-current, transverse phase advance of the CCL and SCL design lattice is shown in Fig. 2. Most SCL cells have phase advances $\geq 60^{\circ}$, while half of the CCL 


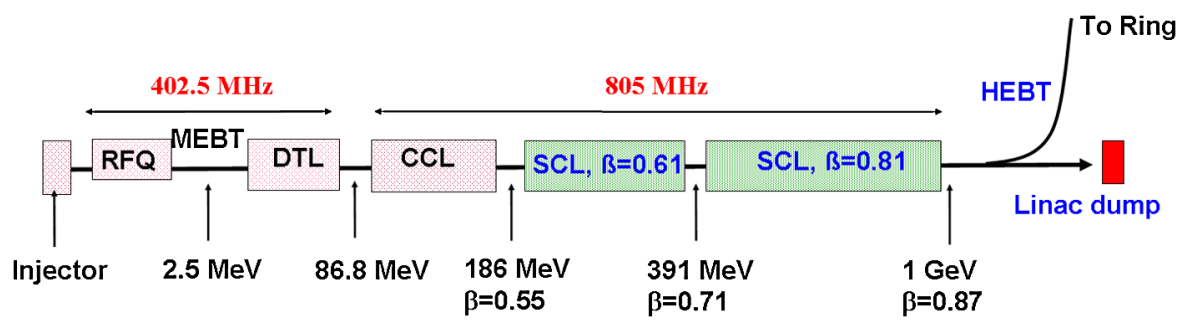

FIG. 1. (Color) SNS linac lattice. Normal conducting section includes injector, RFQ, MEBT, DTL, and CCL; superconducting section includes medium beta $(0.61)$ and high beta $(0.81)$.

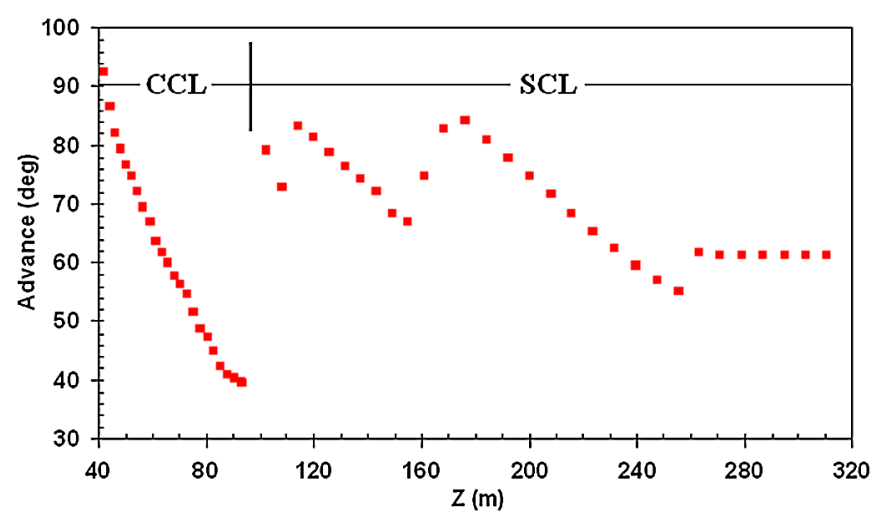

FIG. 2. (Color) Cell-by-cell zero-current transverse phase advance of the CCL and SCL baseline design lattice. Half of the CCL lattice is below $60^{\circ}$, while most SCL cells $\geq 60^{\circ}$.

lattice phase advance lies below $60^{\circ}$. The MEBT lattice is not a standard FODO configuration and the DTL has a permanent magnet periodic lattice of focusing quadrupole, focusing quadrupole, drift space, defocusing quadrupole, defocusing quadrupole, and drift space structure, so they are not shown.

Field components of the CCL and the SCL quadrupoles are measured with rotating coils. The measurements also show the effects of additional dipole corrector windings. The dodecapole components $(m=6)$ have strengths of 30 to 60 units from the quadrupoles, and sextupole components $(m=3)$ are approximately 300 units (the sum of normal and skew terms) from the SCL dipole correctors, as listed in Table I. One unit is the fractional strength $1 \times$ $10^{-4}$ of the quadrupole strength at a reference radius.
These nonlinear multipole components may generate beam halo and cause beam loss in the accelerator systems.

We use the particle tracking code ORBIT [4] to simulate a double lattice similar to that of the SCL dummy section where no rf cavities exist. First, besides the quadrupole dodecapole components, all other nonlinear forces are excluded, in particular rf gap and space-charge effects. The dummy section has nine doublet cells; each cell is $7.89 \mathrm{~m}$ long with quadrupole center-to-center distance $0.92 \mathrm{~m}$. In the simulations we use an 18-cell doublet lattice for a total length of about $140 \mathrm{~m}$.

We find that the sextupole components create emittance growth, but not enough to cause significant SCL beam loss. This result follows from the fact that sextupole strength is proportional to dipole strength and, in routine operation, the dipole strengths are minimal. But the contributions of dodecapoles are quite different. Consider the case of a transversely mismatched beam shown in Fig. 3. We use a mismatch factor of 0.2 , producing a maximum beams size about 1.2 times larger than the matched case [5]. In the simulation the maximum emittance increased fivefold while the rms emittance changed only about $1 \%$. For comparison the matched case is also shown in Fig. 3, where maximum emittance is preserved.

In the design lattice, transverse beam acceptance of the SCL is 50 to $60 \mathrm{~mm}$ mrad, and that of the CCL is approximately $20 \mathrm{~mm}$ mrad. When the maximum emittance increases 3 times in the SCL, as it does in the case of dodecapole effects for mismatched beams, there are beam losses in the superconducting linac. But in the matched case of this simulation, the dodecapole force is reduced by a factor of about 2.5 (5th power of 1.2) because of the smaller maximum beam size. Additionally, effects of

TABLE I. Multipole components of the linac magnets.

\begin{tabular}{lcccc}
\hline \hline $\begin{array}{l}\text { Multipole } \\
\left.\text { (units: } 1 \times 10^{-4}\right)\end{array}$ & $M=6$ & $\begin{array}{c}m=3 \\
\text { From quadrupoles }\end{array}$ & $\begin{array}{c}m=3 \\
\text { From dipole }\end{array}$ & $\begin{array}{c}\text { Reference radius } \\
(\mathrm{cm})\end{array}$ \\
\hline CCL & 38.0 & 3.7 & $\ldots$ & 1.3 \\
& $(0.1)^{\mathrm{a}}$ & $(2.9)^{\mathrm{a}}$ & & 3.0 \\
SCL & 28.9 & -6.9 & 193.5 & \\
& $(4.2)^{\mathrm{a}}$ & $\left(-1.3^{\mathrm{a}}\right.$ & $(207.9)^{\mathrm{a}}$ & \\
\hline \hline
\end{tabular}

${ }^{\mathrm{a}}$ Skew term. 


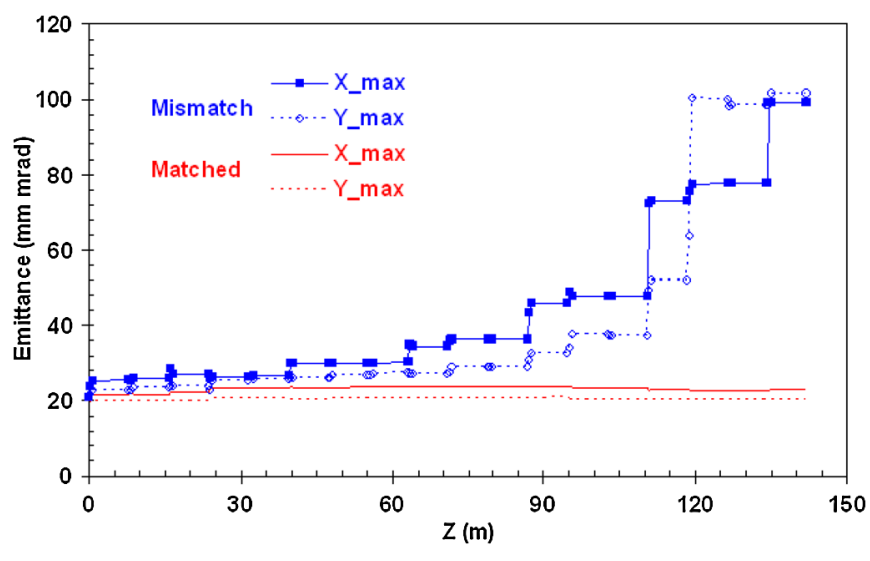

FIG. 3. (Color) Maximum beam emittance dilutions for matched and mismatched beams in an 18-cell doublet lattice with all the doublets made up of identical quadrupoles.

dodecapole forces of the two identical doublet quadrupoles partially cancel, since their polarities at the two quadrupoles are opposite. In the real machine, however, one may not achieve a perfect beam matching, particularly for those large amplitude halo particles. Moreover, the higher-order multipoles of the quadrupole magnets may be worse than the bench test, and different from one another, for they also depend on the magnet assembly detail.

To control the dilution of beam emittance, dodecapole strength is reduced from 30 units to 10 in the simulations, and no significant emittance growth is observed, even in the mismatched case. This result indicates that the dodecapole components of the SCL quadrupole magnets can cause beam loss for particles that are pushed to large radial extent for some reason. In further simulation studies with the ORBIT code, we found that there is a weak resonance in the lattice with a $60^{\circ}$ phase advance. This resonance appears only when the contributions of dodecapoles are significant. In Fig. 4, the maximum beam emittance dilutions versus zero-current phase advances are plotted for

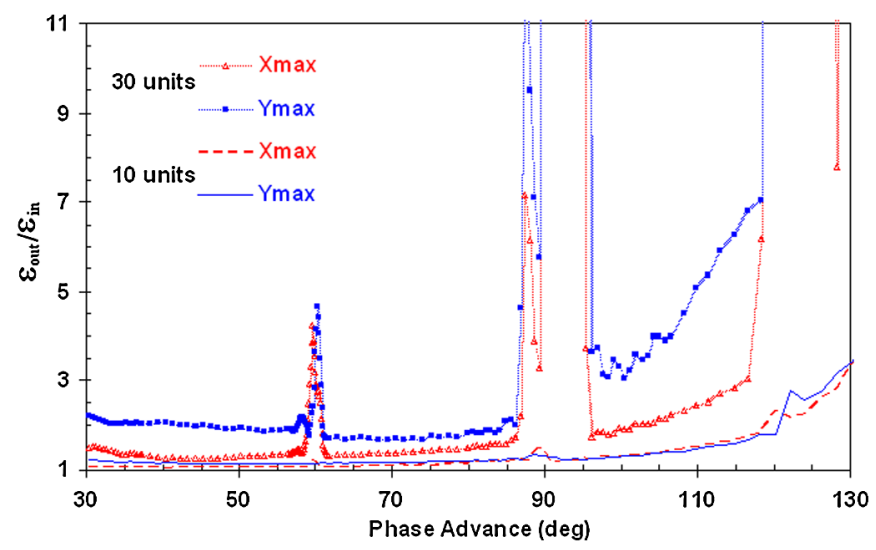

FIG. 4. (Color) Maximum beam emittance dilution versus transverse phase advance for the doublet lattice made up of identical quadrupoles with 30 and 10 unit dodecapoles. two different dodecapole amplitudes: 10 and 30 units. There are three peaks identified at $60^{\circ}, 90^{\circ}$, and $120^{\circ}$ phase advances, respectively. They correspond to the 6th, 4 th, and 3rd order resonances. No other modes, such as the 5 th, 7 th, or 8 th order $\left(72^{\circ}, 51.4^{\circ}\right.$, and $\left.40^{\circ}\right)$, are found in the simulations. The transverse resonance at $90^{\circ}$ phase advance due to dodecapole terms in a FODO lattice was reported in Ref. [6]. To the best of our knowledge, the weak $60^{\circ}$ resonance due to dodecapole components has not been investigated.

\section{HALO GENERATION AND BEAM LOSS}

For particle tracking simulations with dodecapole fields and other nonlinear forces, such as rf gaps and spacecharge, the PARMILA [7] code is used. The simulations start from the MEBT, and use a fully matched $38 \mathrm{~mA}$ injection beam. We first study the linac baseline design lattice without any errors or misalignments. Without dodecapole fields, the maximum beam size is much less than the beam aperture as shown in Fig. 5, and there is no loss in the superconducting linac from the simulation. Next, dodecapole components are added to the linac quadrupoles. In bench tests the average dodecapole amplitudes of all the SCL quadrupole magnets are 29.8 units (the sum of normal and skew terms), with a maximum of 34.1 units and a minimum of 24.8 units. However, the actual strengths could be worse in the linac because of installation imperfections. We use random dodecapole strengths with a mean of 29.8 units, and a standard deviation approximately 5\% exactly the same as the bench tested value, for all the SCL doublets to simulate the more general cases when they are different from each other.

A total of 329 particles are lost in the SCL from 12 simulation runs with different random seeds; each simulation had approximately $86 \mathrm{k}$ injection macroparticles. This gives an average of about $3 \times 10^{-4}$ fractional beam loss in the SCL, as shown in Fig. 6, for well-matched injection beams and the perfect design lattice except the dodecapole

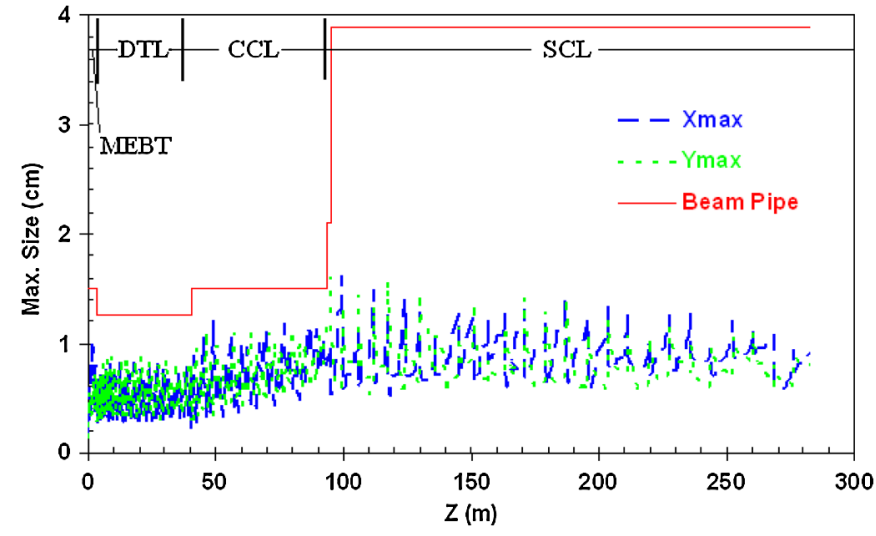

FIG. 5. (Color) Maximum beam size in PARMILA simulation versus the linac aperture, for a $38 \mathrm{~mA}$ design injection beam and the baseline linac design lattice without dodecapole. 


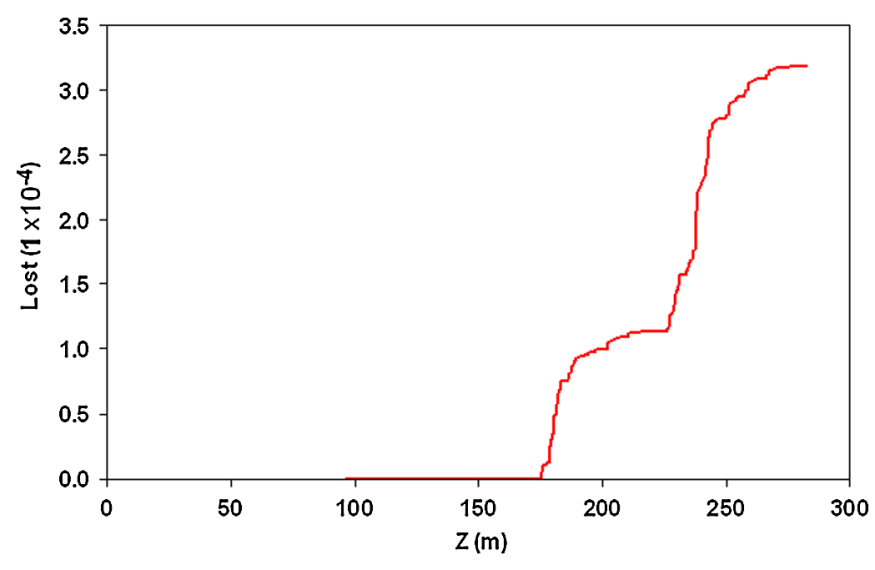

FIG. 6. (Color) Accumulated beam loss in the superconducting linac for $38 \mathrm{~mA}$ design beams and baseline linac lattice without error or misalignment, except dodecapole components.

components. Among the 12 simulation cases, the total factional loss varies from $2 \times 10^{-4}$ to $4 \times 10^{-4}$. The specific loss in each run is presumably determined by the number of large amplitude particles and strength of the dodecapole components of the SCL doublets. Beam halo formation induced by the dodecapole force is also observable in the simulations. Figure 7 shows simulated beam profiles in the SCL dummy section, with and without dodecapole components in the linac. With the nonlinear forces larger amplitude tails (or beam halo) are formed.

We did not include any mismatch, element error, or misalignment in the beam loss simulations of SCL. These results show that, even after a perfect beam match-

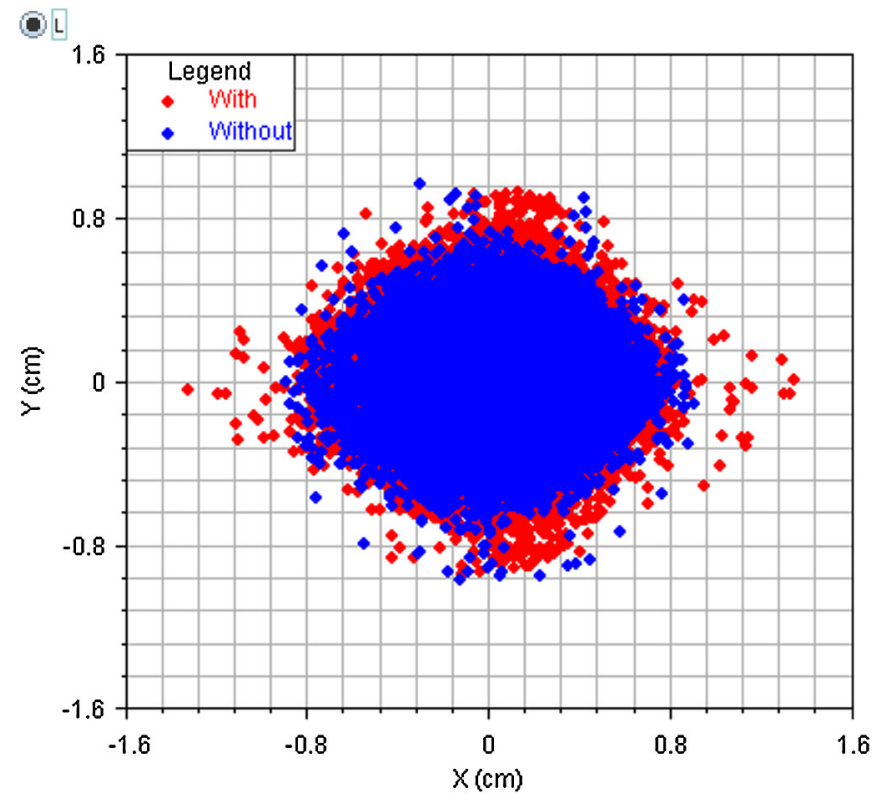

FIG. 7. (Color) Transverse beam profile at the SCL dummy section, for the design linac and the $38 \mathrm{~mA}$ design beams, with and without dodecapole components in the linac quadrupoles. ing, and elimination of all linac errors, save for the dodecapole fields, beam losses well above that anticipated in the design could not be avoided. Thus, within the simulation, the only cause of the beam losses could be the dodecapole fields. Of course in the real machine mismatch, element error and misalignment as well as other effects may cause beam emittance increase as well, and could also contribute to beam loss.

In our experiments, the SCL beam loss is not very sensitive to transverse matching. We did not observe any significant loss difference in SCL with mismatch factors varying from approximately 0.1 to 0.4 . Because the $\alpha$ and $\beta$ functions of a large amplitude halo could be different from those of the beam core, beam matching may improve the core without necessarily improving the halo. Because of limitations in our computer models and performance, we do not have very precise solutions to halo dynamics on the order of $10^{-4}$ of the total beam. Therefore, the most convincing proof of this weak resonance could be from experimental beam loss measurements.

There is a puzzle that has not been solved in our simulations: we do not understand why there are notches in the resonances near $60^{\circ}, 90^{\circ}$, and $120^{\circ}$ phase advances (see Fig. 4). They might come from horizontal-vertical coupling, or the partial cancellation of dodecapole kicks in the two quadrupoles of a doublet, or merely a statistic effect of the small number of large amplitude particle in the simulation, or other unknown factors. Because our current computer models could not provide any more information, to have a better understanding further investigations are necessary in the future; in particular, once a model for this special purpose becomes available to us.

\section{DEDICATED BEAM EXPERIMENTS}

It is surprising to discover that a sixth-order weak resonance at $60^{\circ}$ phase advance induced by a multipole component could cause beam loss in a single-pass superconducting linac with a total length of only about $230 \mathrm{~m}$. However, simulation studies with two different computer models suggest this to be the case, no matter if there is space-charge or not. The predicted fractional beam loss of $10^{-4}$ is beyond the precision of the current model and the capability of an accurate direct beam current measurement. Therefore, we designed two experiments based on the loss measurements by using all the linac beam loss monitors. The experiments were done using the SNS SCL linac set to several different phase advances. The zero-current phase advances of $50^{\circ}, 60^{\circ}$, and $70^{\circ}$ of the SCL lattice are shown in Fig. 8. For a $38 \mathrm{~mA}$ injection beam, simulations with IMPACT [8] yield space-charge depressed phase advances of approximately $30^{\circ}, 40^{\circ}$, and $50^{\circ}$, respectively-which are also shown in the figure. To compare with experimental results, we ran PARMILA simulations for the three cases, shown in Fig. 9. Beam loss caused by dodecapole is the worst for the $60^{\circ}$ phase 


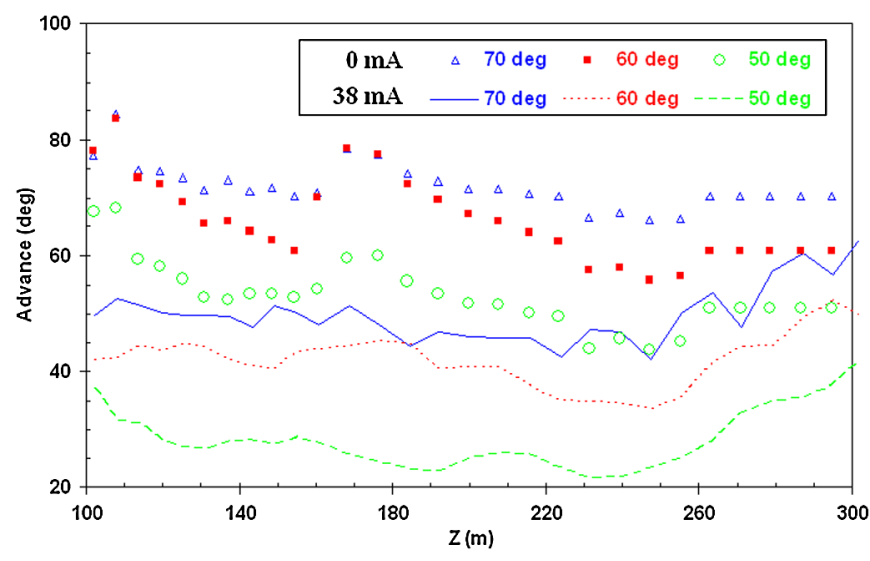

FIG. 8. (Color) Zero-current SCL phase advances at $50^{\circ}, 60^{\circ}$, and $70^{\circ}$ (marks), designed for the beam loss experiments; at $38 \mathrm{~mA}$, space-charge depressed phase advances are $30^{\circ}, 40^{\circ}$, and $50^{\circ}$ (lines).

advance, which is the baseline design lattice. In comparison, the $70^{\circ}$ lattice may slightly reduce the loss, but the $50^{\circ}$ lattice provides a much larger loss reduction.

The beam loss measurement results are shown in Fig. 10. In comparison with the $60^{\circ}$ baseline design lattice, the $70^{\circ}$ lattice reduces the beam loss by approximately $10 \%$, which is very marginal, but the $50^{\circ}$ lattice reduces the loss by $50 \%$. These measured results agree reasonably well with the model predictions. Because of limited physics in the models, errors of the measurements, and other factors not included in these simulations, we do not expect the prediction of the loss level at $10^{-5}$ to $10^{-4}$ to be very precise.

The $70^{\circ}$ lattice cannot cleanly avoid the weak $60^{\circ}$ resonance because of space-charge tune depressions in the high intensity $\mathrm{H}^{-}$linac; the depressed phase advance is approximately $50^{\circ}$ for the production beam, as shown in Fig. 8. This lattice also increases the absolute strengths of dodecapole fields and other nonlinear components with the increased quadrupole strengths. If the beam loss is domi-

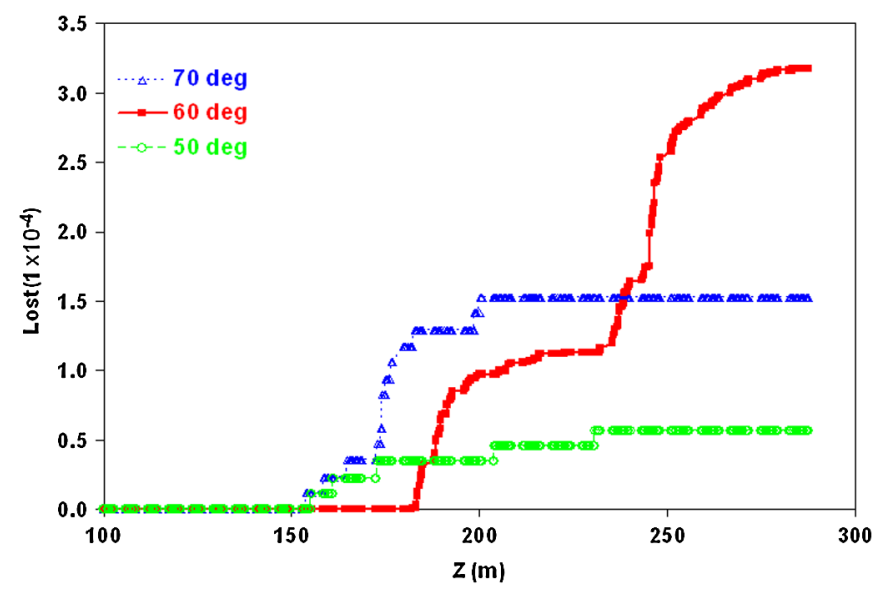

FIG. 9. (Color) Model predicted SCL particle losses for the $50^{\circ}$, $60^{\circ}$, and $70^{\circ}$ zero-current phase advance lattices.

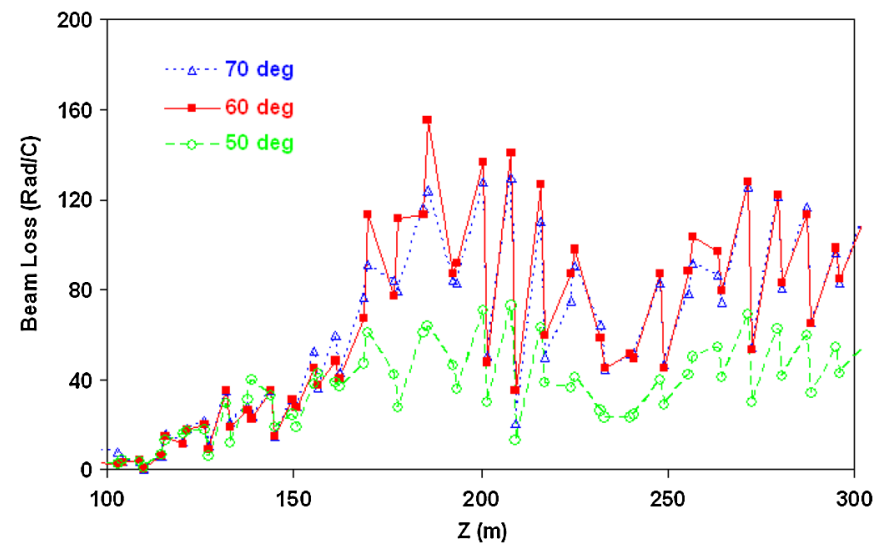

FIG. 10. (Color) Linac beam loss monitor measured SCL losses for the zero-current phase advance $50^{\circ}, 60^{\circ}$ and $70^{\circ}$ lattices.

nated by other factors, such as longitudinal beam halo or beam matching conditions, we should observe a larger loss with the increased quadrupole strengths. However, the SCL loss at $70^{\circ}$ phase advance is slightly reduced compared to the $60^{\circ}$ case. From this experiment, it is demonstrated that dodecapole components can be one of the beam loss factors, especially in the second half of the SCL; the sixthorder weak resonance at the $60^{\circ}$ phase advance has a measurable effect on the SNS beam loss. In the simulation studies, a reduction of dodecapole strength from 30 to 10 units completely eliminates the SCL loss for all three phase advances.

To demonstrate dodecapole resonance more clearly, we turn off all the superconducting cavities, set all the SCL dipole correctors to zero, transport $186 \mathrm{MeV} \mathrm{H}^{-}$beams of $27 \mathrm{~mA}$ through the pure doublet lattice, and measure the total SCL beam loss versus zero-current transverse phase advance. In this experiment, the dodecapole fields are the only dominant nonlinear forces, and they exclude most all other nonlinear issues such as rf gaps, space-charges, and sextupoles. The results are shown in Fig. 11. There are two

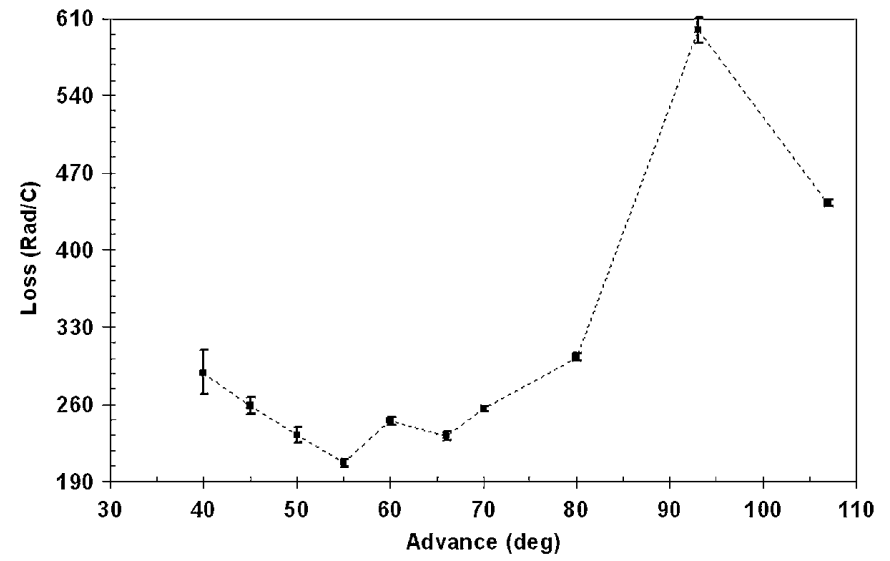

FIG. 11. Total SCL beam loss versus zero-current transverse phase advance with all SCL cavities off, and all dipole correctors at zero. 
peaks identified in the measurements: at $60^{\circ}$ and $90^{\circ}$, respectively; and it is also noted that the $60^{\circ}$ (6th order) resonance is quite weak compared with the $90^{\circ}$ (4th order) one, as having been predicted from the simulations.

\section{CONCLUSIONS}

The SNS linac quadrupole magnets have unfavorably large dodecapole components; and they generate a sixthorder weak resonance around $60^{\circ}$ phase advances in the superconducting linac. According to simulations, the weak resonance leads to beam halo and can cause linac beam loss in addition to several other possible mechanisms. A slight reduction of quadrupole strengths and transverse phase advances from $60^{\circ}$ could avoid this weak resonance and decrease the linac beam loss. The simulation results are verified by dedicated experiments of beam loss measurements.

\section{ACKNOWLEDGMENTS}

The authors would like to thank A. Aleksandrov for many fruitful discussions. This submission was sponsored by a contractor of the United States Government under Contract No. DE-AC05-00OR22725 with the United States Department of Energy.

[1] S. Henderson, Proceedings Utilization and Reliability of High Power Proton Accelerator, HPPA (NEA, Daejeon, 2004), p. 257.

[2] J. Galambos, Particle Accelerator Conference, PAC'09.

[3] Y. Zhang et al., Particle Accelerator Conference PAC'09 (Ref. [2]).

[4] J. Galambos et al., ORBIT User Manual, SNS, ORNL (1999); J. Holmes et al., ICFA Beam Dynamics News Letter 30, 100 (2003).

[5] T.P. Wangler, RF Linear Accelerators (Wiley-VCH, Weinheim, 2008), p. 223.

[6] H. Braun et al., in Proceedings of the Particle Accelerator Conference, PAC'91 (IEEE, San Francisco, 1991), p. 1845.

[7] H. Takeda, PARMILA User Manual, LANL, LA-UR-984478 (2000).

[8] J. Qiang et al., J. Comput. Phys. 163, 434 (2000). 